\title{
Concepts and applications of the Flanders Hydrological Model environment
}

\author{
Thomas Vansteenkiste $^{1, a}$, Joost Dewelde ${ }^{1}$, Pieter Cabus ${ }^{2}$, Inge de Jongh $^{2}$ and Kris Cauwenberghs ${ }^{1}$ \\ ${ }^{1}$ Division Operational Water Management, Flanders Environment Agency, Koning Albert-Il laan 20 bus 16, 1000 Brussels, Belgium \\ ${ }^{2}$ Division Operational Water Management, Flanders Environment Agency, Raymonde De Larochelaan 1, 9051 Ghent, Belgium
}

\begin{abstract}
Today, water authorities face a set of water management challenges, related to new European policies, climate change adaptation, land use planning, urban wastewater and storm water management. In response to these evolutions and anticipating on fast changing IT-technologies, Flanders Environment Agency has developed a set of next generation tools for operational hydrology. These tools are flexible, open and integrate all major components of the water system (principle toolbox). Rather than just providing a set of tools, the toolbox is integrated into existing information systems by the latest technologies using open standards and web protocols. Currently, the toolbox is used to establish a regional hydrological model set for Flanders. This large scale model will produce information on the hydrological status (runoff, subflow, infiltration, soil storage, etc.) at any location in Flanders. Furthermore, an interactive web-application of the toolbox was developed to enable hydrological modelling through the internet. Besides the fulfilment of the modelling needs of the Flanders Environment Agency, the initiative aims to foster collaboration, not only between governmental agencies with operational duties in water management, but also between the different communities in support of operational hydrology.
\end{abstract}

\section{Introduction}

Water authorities need robust simulation models to support the management of their water resources. These models help to estimate the impact of planned infrastructure works, to maintain water supplies, to make projections of the water system under changed conditions (climate and land use), to design flood protection measures, to forecast floods, etc. In the late ' 70 and ' 80 there was proliferation of hydrological models, among others PDM [1-2], NAM [3], MIKE SHE [4-5], SWAT [6], TOPMODEL [7] and IHACRES [8]. Some of these models are currently in operational use by water authorities. Examples include the Grid2Grid model at the UK flood forecasting centre [9], PDM in the Environment Agency National Flood Forecasting System [2] and the Flood Forecasting system of unnavigable rivers in Flanders [10-11], while the NAM hydrological model is applied to forecast floods for Flanders' navigable water courses [12-13]. Model application by water authorities is often subject to the common practice, and therefore based on one specific model. Rarely an objective model selection seems to be conducted. However, the type of model code and structure might strongly affect the simulation results [a.o 14-19]. Although hydrological models have similar characteristics, they apply different schematizations and data affecting their simulation results. Depending on the type of application, the catchment characteristics and the data availability, a different model code should be considered. Additionally, the use of model codes is often limited to the administrative level and detail within their juridical responsibility and they are applied on local project scale. Given different codes might generate inconsistent results, this might impact management decision across organizations. In contrast to other countries, there is currently no region-wide simulation model available in Flanders for applications like flood mapping and forecasting. Across the UK a distributed runoff model is nationally used in the flood forecasting system [20]. In the Netherlands a National Hydrological Instrument (NHI) was developed to support both longterm policy analysis and daily water management [21]. One reason for lacking a regional model is Flanders long tradition of modelling. Over the past decades operational processes and modelling instruments at the different scales in Flanders have organically grown towards closed and complex chains in which the models are strongly embedded. Each model within the chain was developed only for a specific objective. This limits not only the reuse of the models across water disciplines and organizations, but also slows down the innovation of the operational chains through science (data assimilation, radar input, probabilistic forecasting, etc.) and technology (mapping and charting tools, web services, etc.).

In response to these findings and anticipating on fast changing IT-technologies, the Flanders Environment Agency has taken an initiative to build and maintain an

\footnotetext{
a Corresponding author: t.vansteenkiste@vmm.be
} 
enhanced standardized platform for seamless modelling the Flanders' hydrological system, namely the Flanders Hydrological Model environment, also FHM environment. The model environment comprises a set of model codes and tools, that are flexible, open and integrate all major components of the water system (principle toolbox). The modelling environment is open, which allows for flexible and harmonized workflows with other models or existing information systems like meteorological databases, river models and dataassimilation tools. This paper will outline the modelling environment and highlight some of its applications. The overview starts by describing the goals and potentials of the environment, followed by a short description of the methods, models and data used by the environment and the connectors linking them. In a next section two applications are illustrated. The first one deals with an application of the modelling framework over Flanders. Each site in Flanders - gauged or ungauged - can be modelled by the framework allowing authorities to generate maps on the water status for any location of interest. The second application concerns the integration of the framework in a common web service infrastructure. This allows for online modelling through a browser and for sharing and re-use of models. The modelling environment is currently being developed and expanded. A conclusion provides an outlook to future directions and developments.

\section{Goals and potentials of the Flanders Hydrological Modelling Environment}

For what concerns a better and more integrated management of water resources and analysis of water data in Flanders, Flanders Environment Agency strongly believes that a transformation of their modelling data, tools and instruments towards open data, less fragmentation and more standardization is needed. Hence, on the long term the Flanders Environment Agency wants to transform and extend its operational monolithic - modelling systems and forecasting chains into layered, web-enabled, flexible, modular, open and standardized components and services.

A first step towards this ultimate goal is the development of a standardized platform for modelling the Flemish hydrological system. The platform was built following the latest trends in science and technology (e.g. web services, information layers, model interoperability, flexible workflows, etc.). The modelling environment was set up with several objectives in mind. A key objective of the model environment is to build a spatially and temporally accurate hydrological model of Flanders that might, in contrast to current operational models, have a common basis and simulate in a consistent way the hydrological processes over Flanders. This calibrated model aspires to perform a role in water management across Flanders by delivering modelling products (simulations, impact results, forecasts, flood maps, etc.) as reference or authentic water information layers. That way the model might contribute to a more consistent model support for policy making across different administrative levels. Where models are based on common data sources, data processing principles and model codes, discussion will be avoided [21]. As a second objective the modelling environment has to offer a framework which enables more innovative developments. This must on the one hand guarantee an enhanced hydrological model performance and on the other hand allow for a better integration of hydrological models into services useful for broader applications such as hydraulic inundation modelling, forecasting, etc. For example, it might function to assess the impact of any kind of flood. In combination with other models, the modelling environment will enable an agile identification of the location and consequences of forecasted floods. These results could then be used by other information platforms for emergency purposes and crisis management.

The Flanders Environment Agency is however aware that these hydrological modelling and water engineering challenges require collaboration and transdisciplinary exchange. An additional objective of the environment is to enable more structural collaboration between science and operational services in order to continuously bring new innovations into the water business and subsequently further enhance the performance of simulation models. A suitable organization structure should be developed to support this collaboration. Cooperation within a hydrological community of local and more regional water modellers, but also with technology partners and universities to improve the modelling tools and create a common ground for modelling, will lead to a more effective use of the modelling environment and a higher performance. The entire community of researchers, modellers and model developers will benefit from such an environment for modelling.

\section{Concepts of the Flanders modelling environment}

\subsection{General overview of the Flanders Hydrological Modelling Environment}

The Flanders Hydrological Model environment provides a set of functionalities that work together to build, calibrate, analyse and evaluate hydrological models. It enables to:

- define lumped and distributed hydrological models, and provide them with input time series and radar images using default links with meteorological and spatial databases,

- calibrate the models to the observations by a set of calibration functions using graphs and statistics,

- simulate and evaluate the outputs of the models, including state variables.

This hydrological modelling framework is the core of the FHM environment. It provides flexibility, such that one can redefine or add extra modules to enhance the model performance, while retaining as much of the default framework as useful. Examples include new soil moisture 
accounting models, other routing methods, additional calibration routines, multiple objective functions, more evaluation statistics. To achieve the modelling framework functioning it needs to be fed with input. The framework is connected to existing meteorological and spatial databases. Time series of rainfall and evapotranspiration are extracted from a central meteorological database using open standards and protocols. This enhances the transparency and reproducibility of the modelling process, and avoids duplication of data storage. Simple functions within the modelling environment allow the users to define the configuration files for this times series transfer from the database to the modelling framework. To better account for the location and intensity of the rainfall, the modelling framework can make use of high resolution, gridded radar measurements and forecasted rainfall data. This is particularly useful for event based flood modelling. Functionalities are also foreseen within the FHM environment to recalibrate raw radar images with ground-based measurements of rainfall. These are based on the methodology of Martens et al. [22] and serve to better correspond the radar observations to the ground-based measurements. In case of distributed modelling, the spatial information is provided by reference datasets within the FHM environment. These data are derived from national databases and include maps of soil type, land use and ground elevation. Figure 1 shows the major input and output flows between the model framework and the databases. A brief overview of every component is presented in the following sections.

The FHM environment is an open modelling environment and was built within the PCRaster-Python framework. Python is an interpreted, object-oriented, extensible and free distributed programming language. Its simple syntax makes it very attractive. However, to make it useful for the construction of a modelling environment, additional spatio-temporal functionality is necessary. The PCRaster Python Extension [23] has taken this functionality from the PCRaster software [24], which is a script framework that can be used for interactive visualization. Typically to Python model development applications are the modular composition of the model. As the Python code is available under an open source licence, users always have the option to adapt modules of the FHM environment as required. Hence, the modelling environment can grow and evolve. That way the modelling environment might also be tailored to all kind of needs or it can be used cohesively with workflows based on popular software in broader applications. The cost of maintenance and developments of the environment should on the long term be shared by a hydrological community, representing academics, water authorities and consultants in Flanders.

\subsection{Central hydro-meteorological database}

A central and unified meteorological database, managed by the Flanders Environment Agency, provides the rainfall and evapotranspiration input and flow data for the hydrological model applications. The database includes monitoring data of 43 rain gauges, 8 meteorological stations recording all parameters related to evapotranspiration (a.o. radiation influx, ground irradiation, soil temperature, air temperature at different heights), over 150 hydraulic structures with their up- and downstream water levels, gate or weir positions and more than 200 river gauges of which 94 also register discharges (Figure 2). Besides these basic data sources, the database also stores additional parameters (e.g. soil moisture) and derived data products (e.g. catchment interpolated raw and recalibrated radar rainfall, time scale aggregations, Thiessen interpolated rainfall, averages, etc.). All measurements are locally stored and are automatically retrieved by data acquisition software to the database. Standard data calls are executed based on

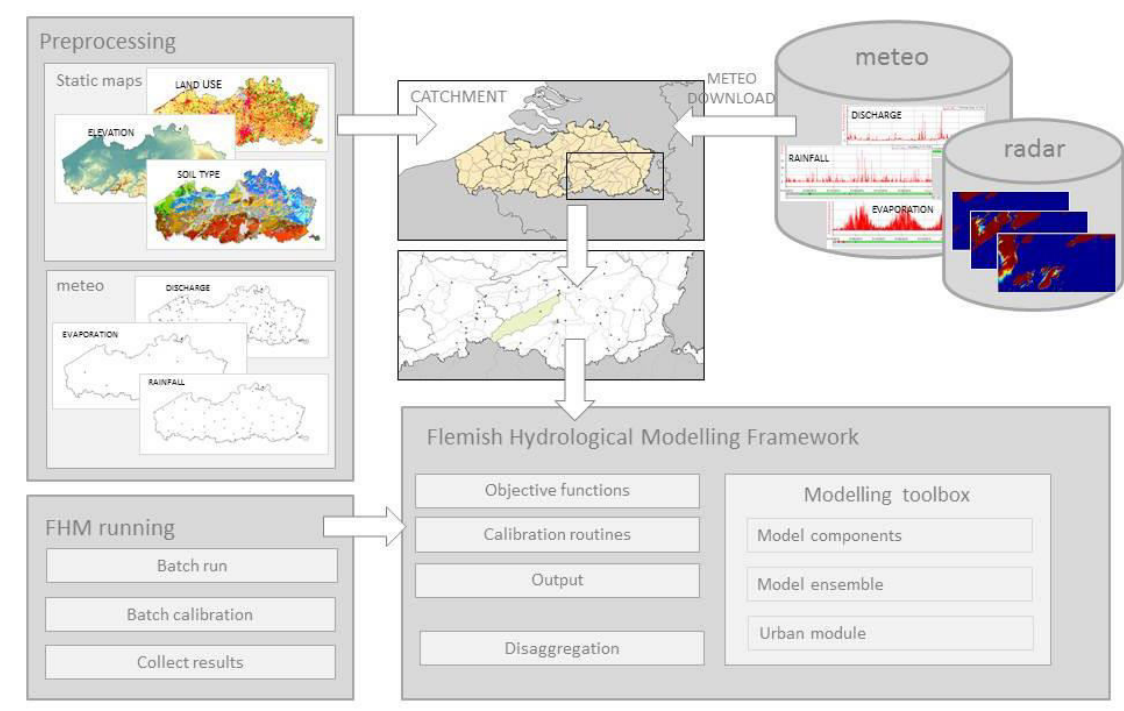

Figure 1: Concepts of the Flanders Hydrological Model environment 


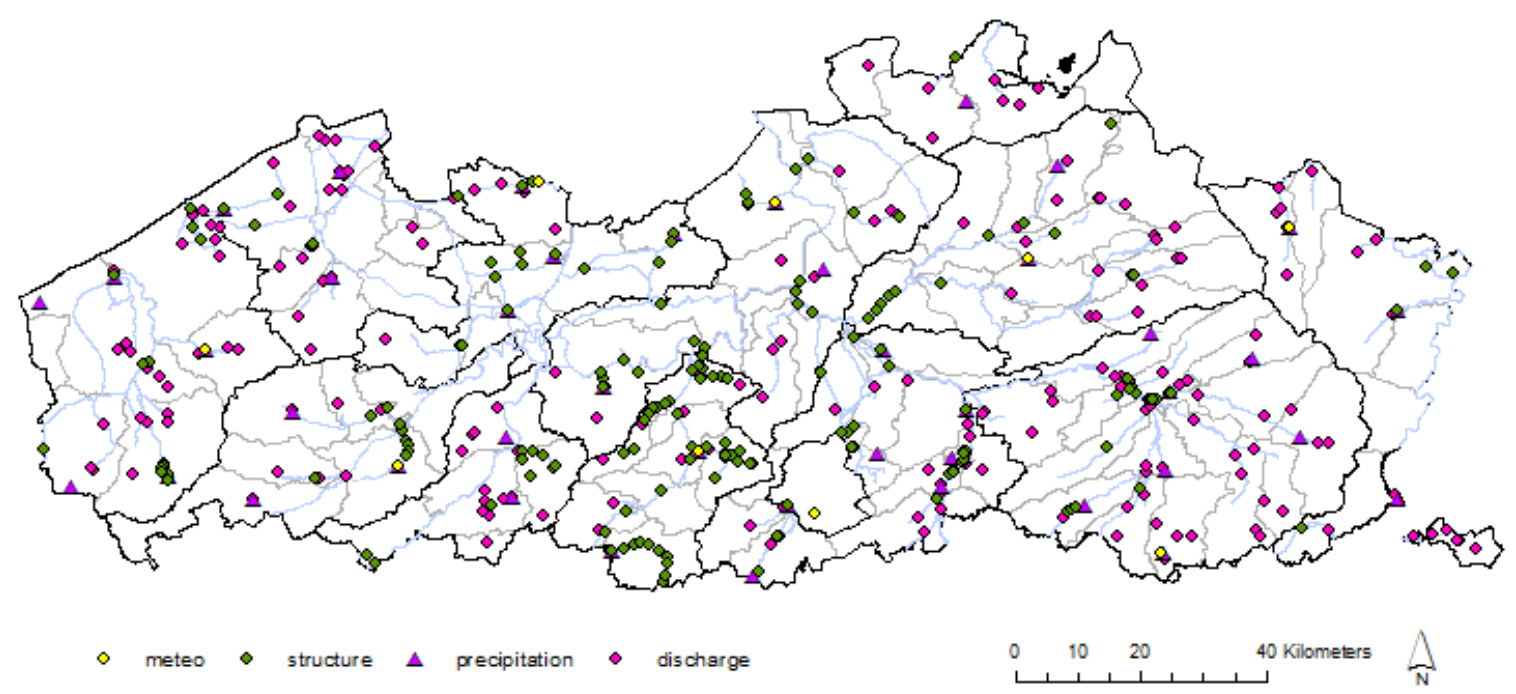

Figure 2: Overview of hydro-meteorological monitoring stations in Flanders

the urgency with a 3 hour to 15 min interval. All these monitoring data are publicly available on www.waterinfo.be. However, the data might also be requested by web service, which are then accessed using standard web protocols. Web services are platform independent and can easily be integrated in workflows for easy data retrieval, manipulation, visualisation, and communication. Hence, the modelling environment is foreseen to access the meteorological database by these protocols. The standard that enables the access consist of three components, and looks like the following: http://www.waterinfo.be/tsmpub/KiWIS/KiWIS?type=qu $\underline{\text { eryServices\&service }=\text { kisters \&request }=\text { getTimeseriesvalu }}$ es\&ts id $=013418042 \&$ datasource $=0 \&$ format $=$ html\&peri od=P3D\&to=2015-03-24T21:00:00+01. The first component shows the service root URL, followed by the resource path. The second component lists the station parameters, while a last part is related to the data format and the time period of interest. Identification codes of the monitoring stations are stored in distinct tables within the modelling environment. For the retrieval of the data series, the unique numerical key of the monitoring station needs to be selected, together with the time feature and output format. Specific functions were designed within the model environment to create the web protocols from this selected information. The uniqueness of the identification keys results in a specific protocol to retrieve the requested data records. This way of working ensures that data, applied by the modelling framework, is harmonized and quality-guaranteed. Another and even greater advantage is that the user can make use of the latest available data from the databases in the models. Next to the times series also station and catchment characteristics, e.g. model area, station coordinates, station numbers, etc., can be consulted through web services. For the set-up of a catchment model, the framework can also search automatically for the records from the most approximate meteorological stations. These records are formatted in such a way that they can immediately be applied in a simulation.

\subsection{Spatial data sources}

Spatial data is only required when a model is intended to run in distributed mode. Maps with elevation, land use and soil type data are the 3 base maps used in the model environment. All data have been extracted from open spatial data infrastructures and are publically available. However, the model also needs information on the river network (slope, length, width, gradient, banks), catchment boundary, drainage network but these data layers are derived from the elevation data during model pre-processing steps. Given the fact that it is quite difficult to identify the exact catchment boundaries and river bed location, in particular in plain areas or regions with lakes or artificial channels, the digital elevation model might be manually adjusted to precisely identify the catchment polygons and river networks. Land use and soil type information are important to represent the spatial distribution of the catchment characteristics. The modeller can freely decide what kind of land use classes and soil types are used in the base map. However, the considered classes must significantly distinguish from each other with regard to their effect on hydrological processes. Each of these classes is then characterized by quantitative attributes via a lookup table. These attributes are used for disaggregation of the model parameters and is further outlined in the next section on calibration.

The Flanders Hydrological modelling Environment makes use of area-wide information. Elevation, land use and soil type information are provided for the entire Flanders region. However, data collection is not limited to the Flanders region, because catchments do not follow the administrative boundaries. The base maps of Flanders are therefore extended with additional data, collected from various sources in Wallonia and Brussels. The chosen resolution of these maps will depend upon the purpose and accuracy requirement of the modelling application. For small water catchment areas, where low discharges are monitored and more detail is needed, a smaller density of grids is recommended. This implies 
that all base maps have to be provided at every relevant resolution.

The preparation of the spatial data for the modelling of a specific catchment implies the identification of the spatial distribution of land use classes and soil types and the determination of the complete drainage structure of the watershed. The model environment provides a set of functions that extracts this information for the catchment from the base maps. The boundaries of the catchment of a selected location of interest are identified based on the drainage network. Built-in functions foresee a fully automated way to cut the catchment area from all relevant spatial base maps. This makes the spatial data preparation of the modelling very straightforward.

\subsection{Hydrological modelling framework}

\subsubsection{Lumped models}

The class of hydrological models considered in the modelling framework are conceptual models. On the one hand the framework relies on already existing and implemented, fixed model codes $[1,3,25]$. These meet the concepts of the current model codes of different operational water institutes in Flanders. On the other hand the framework includes a more modular approach, which allows to combine different model processes [26]. A model is then created by coupling the most appropriate process conceptualizations together in order to assess the most optimal model structure for a given situation (e.g. linking soil moisture accounting from one model, to the routing of another model). However, all model combinations remains parametrically parsimonious and computationally efficient. Others examples of such a flexible or multi-model frameworks are the Modular Modelling System (MMS) [27], the Catchment Modelling Framework (CMF) [28] and Superflex [29].

For calibrating the models to the observed data several functions are foreseen within the framework. The modeller can choose between an optimization based on the shuffled complex evolution algorithm (SCE) [30] or an autocalibration using Latin Hypercube simulations (LHC). In the latter one the results of the parameter sweep are evaluated against multiple objectives. A combined performance measure, based on the statistics of the individual performance measures, is then applied to select the best parameter set. The objective functions for this manner of calibration focus on different aspects of the hydrograph, i.e. overall water balance, shape of the hydrograph, peak flows and low flows. The modeller is able to select the most appropriate one(s) for its application. That way, different calibration schemes and objective functions can be applied for different types of problems.

Model simulations are commonly evaluated against multiple objectives. A classic protocol is followed, including a comparison of the observed and simulated river flows, a set of statistical measures, an inspection of the simulated hydrographs during storm events and a comparative analysis of the water balance. Additionally peak and low flows from the simulation results are compared with the observations in Flood-DurationCurves and QQ-plots.

The modeller could calibrate one specific model structure or prepare batch tests. By activating the batch, the modelling framework will sequentially run or calibrate each predefined model structure. The outcome of this batch test will be the best performing structure and corresponding optimal parameter set. When data limitation restricts the selection process and more than one model structure provides plausible results, a solution can be to use an ensemble of models rather than only one and to interpret the results of the ensemble together to improve the reliability. The framework has a module to support this ensemble modelling for situations where the data accuracy is low and the unpredictability is high (Figure 3).

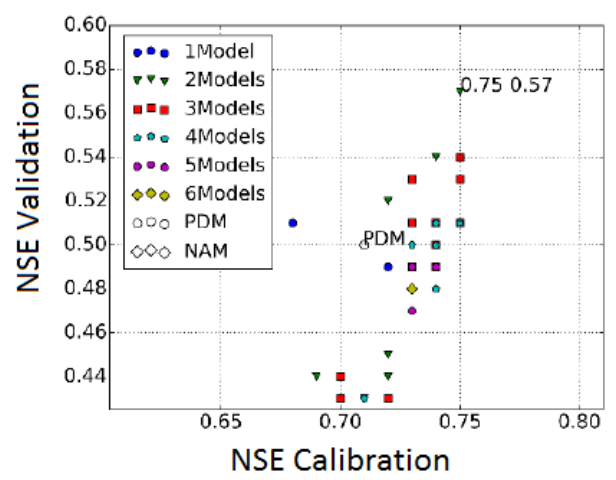

Figure 3: Ensemble modelling result within the FHM-environment

\subsubsection{Distributed models}

To build spatially distributed models, a set of spatial linking features needs to be defined. A two-domain approach is followed, first linking non-river cells to the nearest downhill river cell, and then aggregating all river cells to the outlet cell. For each domain a number of functions are provided. For the land domain a source to sink method, a basic and a detailed implementation of the grid to grid method are foreseen. For river cells linking, the source to sink method and the kinematic wave method were implemented. The parameterization and calibration of the distributed model is based on Quan et al. [31]. Parameters that are calibrated in lumped scale will be transferred to distributed scale. An advantage of this top-down calibration approach lies in the model structure identification. At the lumped scale, the model structure identification and parameter calibration has low uncertainty, given the number of parameters are limited. The spatial disaggregation of the calibrated model parameters at the lumped scale into higher spatial resolution is done by using the maps of elevation, landuse and soil type and its related lookup tables. The distributed parameters are adjusted relatively based on the parameter values from the calibrated lumped model. This means that for each parameter the spatial average is identical to the uniform parameter value, but that relative spatial variations are given to that averaged value, proportional to the geographical catchment characteristics [31]. Given this spatial disaggregation is based on 
assumptions regarding the dependency between model parameters and geographical catchment characteristics, which may vary from region to region, some adjustments may be needed. To account for this, two additional parameters are introduced. The first one allows to increase or decrease the mean value for each parameter, and the second one defines to what extent the spatially distributed values may deviate from the mean value. The distributed model allows to generate spatially variable outputs. These model outputs can be compared to flow data within the catchment, groundwater well heads and soil moisture levels. Next to a comparison of times series at specific point locations, the model output can also be exported as maps. This distributed approach offers the framework a great potential for the modelling of ungauged basins.

Within the modelling environment modellers can determine what the best (combination of) data, models, tools could be for their particular application. As the model code is open the modeller always has the freedom to adapt it as required. He may include additional components to emphasize processes that are significant and/or dominant for his catchment. Additional modules were already created to address specific processes and improve the simulation results such as time delay, snow melt, urban runoff and hydrograph damping to account for upstream flooding.

\section{Applications of the Flanders Hydrological Modelling environment}

\subsection{Flemish Hydrological Model}

Currently, the FHM-environment is used to establish a regional hydrological model set for Flanders, named the Flemish Hydrological Model (FHM). This large scale model will provide information on the hydrological status (runoff, subflow, infiltration, soil storage, etc.) at any location in Flanders. The general purpose of the FHM is to set a hydrological model reference for Flanders, which can be applied within other water related disciplines such as soil erosion, agriculture, water quality management, drought policy, forecasting, etc. Hence, it might become an essential instrument for Integrated water management. The large-scale Flemish Hydrological Model is built up from many sub-models, which are linked within the FHM environment. The base maps for these individual sub-models were foreseen on a raster of 100 by 100 meters, and the temporal scale for the models was fixed at hourly. The sub-models are individually calibrated with the SCEUA optimization modules using the Nash-Sutcliffe objective function [32]. This way of calibration will guarantee a good overall performance of total flows over Flanders. However, for more specific applications like flood or drought modelling, a more detailed calibration might be needed considering additional criteria. All model structures within the framework were evaluated in order to select the most appropriate model structure for each catchment. The parameter calibration was done first for the lumped model and parameter values were afterwards disaggregated to the distributed model. After validation the individual sub-models are combined within the FHMenvironment, where they are linked and contribute to the larger Flemish Hydrological Model. For every gauged site in the Flanders region a distributed model can be calibrated. Also ungauged sites can be modelled, using parameter sets of nearby gauged catchments. Validation of these models might then occur based on groundwater and soil moisture information. However, currently not all catchments over Flanders are being modelled.

Within this paper four sub-models of the FHM are highlighted. They cover the catchments of the Handzamevaart, Zwalm, Barbierbeek and Zwart Water, which are relatively small - between 32 and $114 \mathrm{~km}^{2}$ and represent the different hydro-geological regions of Flanders. Figure 4 illustrates their location in Flanders, while table 1 gives information on the area and the default derived river network used by the model framework.

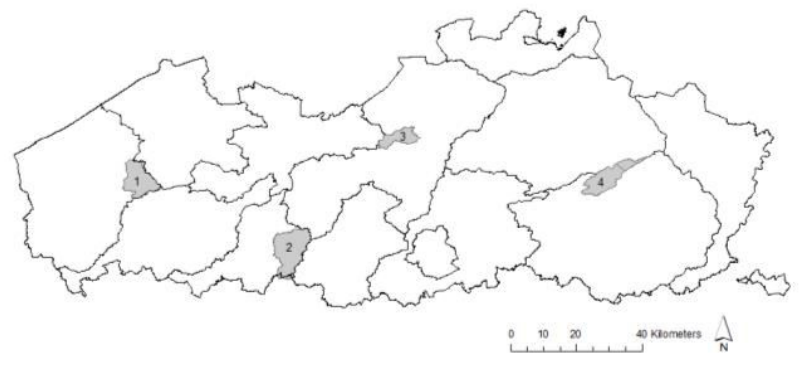

Figure 4: Selected catchments in different hydro-geological region over Flanders

Table 1: Selected catchments with their river network derived from the base elevation map

\begin{tabular}{l|cccc}
\multicolumn{5}{c}{ Table 1: Selected catchments with their river network derived from the base elevation map } \\
\hline Catchment & $\mathbf{1}$ & $\mathbf{2}$ & $\mathbf{3}$ & $\mathbf{4}$ \\
Area $\left[\mathrm{km}^{2}\right]$ & Handzamevaart & Nederzwalm & Barbierbeek & Zwart Water \\
River network & 78.55 & 113.65 & 32.13
\end{tabular}


The ability of a hydrological model to simulate the runoff depends strongly on the accuracy of its input. The quality of the input series was extensively analyzed during the model set-up. The most appropriate series and periods were selected for the model calibration and validation. Hence, the calibration and validation periods may vary between the catchments of FHM. For example, a change of the stage-discharge conversion in march 2013 limits the data consistency at the Nederzwalm, while for the Handzamevaart consistent discharge data was found from 2004 on. Within this paper, the performance of the individual sub-models is presented for a recent period (08/2013-12/2015). It is expressed by a set of basic statistical measures (Nash Sutcliffe Efficiency- NSE, Root Mean Squared Error - RMSE, correlation - R2 and Water Balance error - WBE) (table 2), plots to compare time series (figure 5), the water balance (figure 6) and some winter and summer events (figure 7).

Table 2: Performance statistics of the FHM sub-models

\begin{tabular}{l|cccc}
\hline Catchment & $\begin{array}{c}\text { NSE } \\
{[-]}\end{array}$ & $\begin{array}{c}\text { RMSE } \\
{\left[\mathbf{m}^{3} / \mathbf{s}\right]}\end{array}$ & $\begin{array}{c}\text { R2 } \\
{[-]}\end{array}$ & $\begin{array}{c}\text { WBE } \\
{[\%]}\end{array}$ \\
\hline Handzamevaart & 0.72 & 0.50 & 0.87 & 2 \\
Zwalmbeek & 0.82 & 0.62 & 0.91 & 5 \\
Barbierbeek & 0.80 & 0.19 & 0.90 & 21 \\
Zwart Water & 0.81 & 0.23 & 0.12 & -1 \\
\hline
\end{tabular}

The runoff results between the catchments are quite similar. For all four catchments the models produce runoff series that are in good accordance with the observed data. This is underlined by the statistical performance measures: NSE values are good $(>0.72)$ and correlation coefficients $\left(\mathrm{R}^{2}\right)$ vary between 0.85 and 0.9 , except for Zwart Water. This means that for all other catchments the simulated hydrographs show a good agreement with the observations. From figure 7 , it is noticed this is particularly the case during the winter periods. Summer hydrographs are less well simulated and some of the peaks are missed entirely (a.o. Barbierbeek). This might occur during local convective storms, which are not captured by the network of pluviographs, and whose data is therefore missing in the input rainfall series. In most cases the peak values of the hydrographs are estimated well, although the volumes of the associated hydrographs are often underestimated. This is particularly seen in the results of the Nederzwalm model. The total flow volumes are well estimated. Water balance errors (WBE) are limited to 5\%, except for the Barbierbeek. Considering models remain conceptualizations of reality, it can be concluded that the above calibrated models are able to adequately describe the general hydrological behavior of the catchments, although they show limitations in one or more performance indicators. In a next step, the models will be transferred to contribute to FHM. The first version of the FHM is currently under development. Nevertheless, the modelling environment is envisaged as a modeling framework over the entire Flanders region that ensures continuity of modeling in time and space. As such, it might become a standard support for policy.
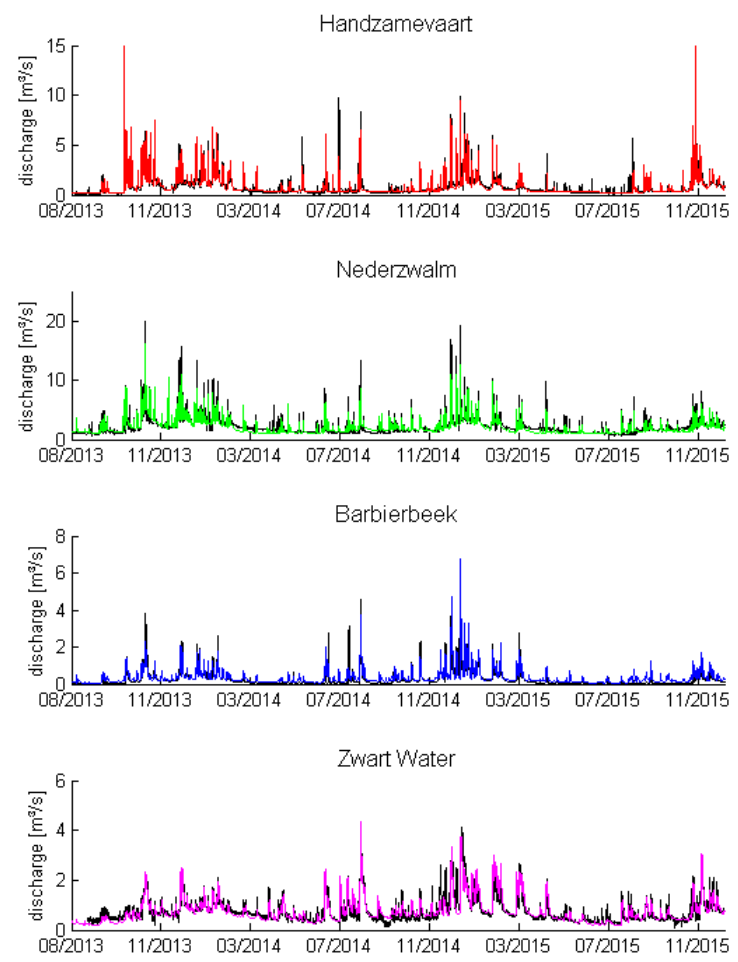

Figure 5: Observed and simulated runoff discharges of the FHM sub-models
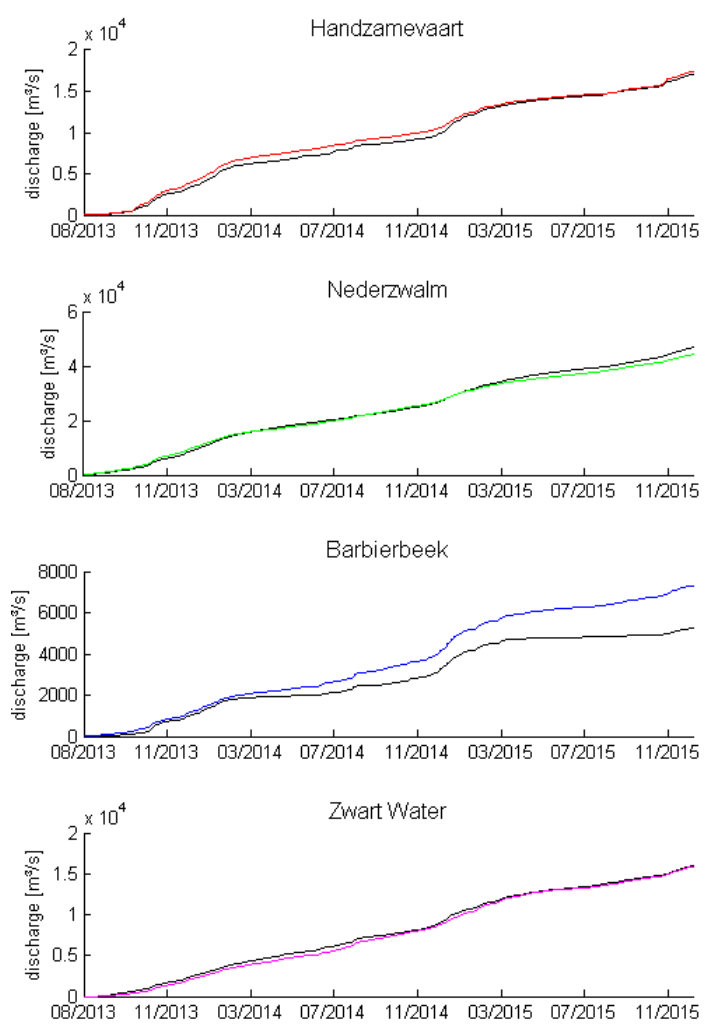

Figure 6: Observed and simulated cumulative discharges of the FHM sub-models 

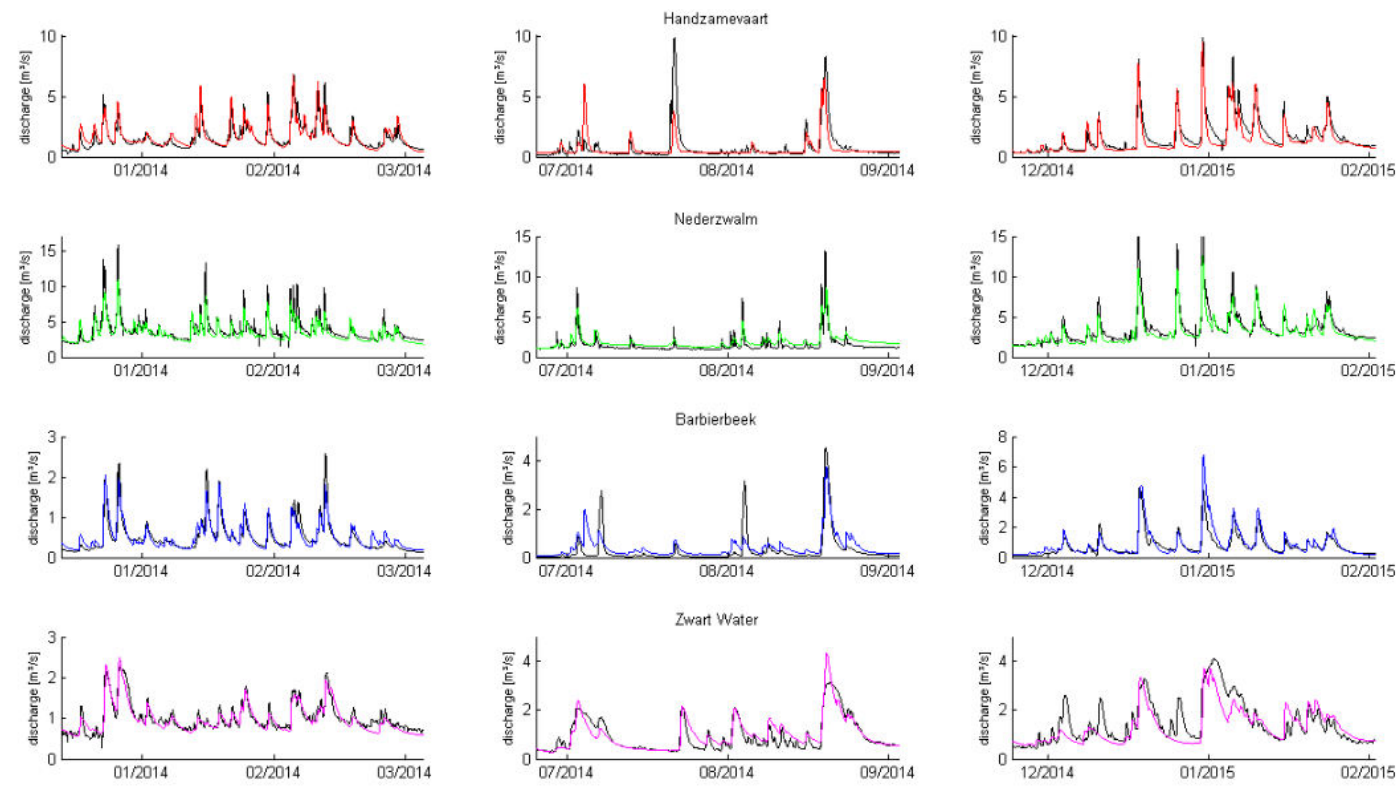

Figure 7: Observed and simulated runoff discharges for winters of 2014 and 2015 and summer of 2014 of the FHM sub-models

\subsection{Modelling as web application}

For its water modelling the Flanders Environment Agency currently makes use of standalone model software, which depends on a particular computing platform and hardware configuration. The software runs on several servers on a local area network. It is also often temporarily - accessible to external consultants and universities during modelling studies. However, this approach brings a number of issues along with respect to the supply of accurate data and data storage capacity, the use of software licenses, the processing of output data, the development of tools and applications, the management of the model database, the overload of requests and user questions. On the long term this way of customer interaction might no longer be efficient. To overcome these limitations on the current modelling systems, the potential of web enabled hydrological modelling was examined. This means databases and hydrological models are run on a server and results are distributed over the internet. This offers several advantages in comparison to the current set up, a.o (i) model output is stored on the local database on the desktop of the customer, (ii) licenses are no longer necessary by the use of open models and data, (iii) time consuming and user specific requests are limited, (iv) exposing hydrological simulation models on the web can make use of a growing number of data services, (v) facilitating the integration with other services and systems. Examples of innovative technologies that already offer such ways of modelling are the EVO portal, www.evo-uk.org, [33], which deploys a web application to model local flooding and diffuse pollution. The Virtual Globe application, http:/virtualglobe.geo.uu.nl [34], provides a platform for configuring and running high resolution environmental models nearly anywhere in the world. Another related example is reported by the
ENVISION project, http://www.envision-project.eu [3536]. It incorporates an environmental decision portal to support the creation of web-based applications enabled for dynamic discovery and visual service chaining.

In this work, an interactive web application of the FHM-environment was developed to make the hydrological modelling framework potentially available to a far broader set of clients. It allows users to simulate the hydrological sub-models of FHM through the internet for any period. At the backend a workflow was constructed between the hydrological modelling framework, the database with the input and calibration data and a workflow manager. The workflow manager, allowing for automatically combining and connecting different data sources, models and web services, was also implemented by Python scripts. In order to test the level of modularity and flexibility of the FHM-environment, additional workflows were created by linking the modelling framework through web protocols with an extra workflow manager (FEWS), the open CMD hydraulic modelling tool [37] and a TELEMAC 2D flood inundation model.

At the front end a user interface to the modelling framework was developed as a case study to examine its integration within web based technologies. The application consists of the following user-interactions, which are also illustrated in figure 8: (i) selection of the basin of interest by its catchment outlet; (ii) selection of the time period to run the model for the selected basin. Based on this user input, observed river flows and precipitation data, as well as pre-calibrated parameters of the user selected model are queried from the hydrometeorological database; (iii) subsequently, the selected model is run with the parameter values and the results are presented in the web interface as tables, maps or charts. The results can be analyzed online using numerical functions. The model can be run over and over for 
different time periods. Details on where and how the data are held are hidden from users. This allows for data to be used in models and simulations without necessarily giving it to the users, avoiding some of the delicate issues of ownership.

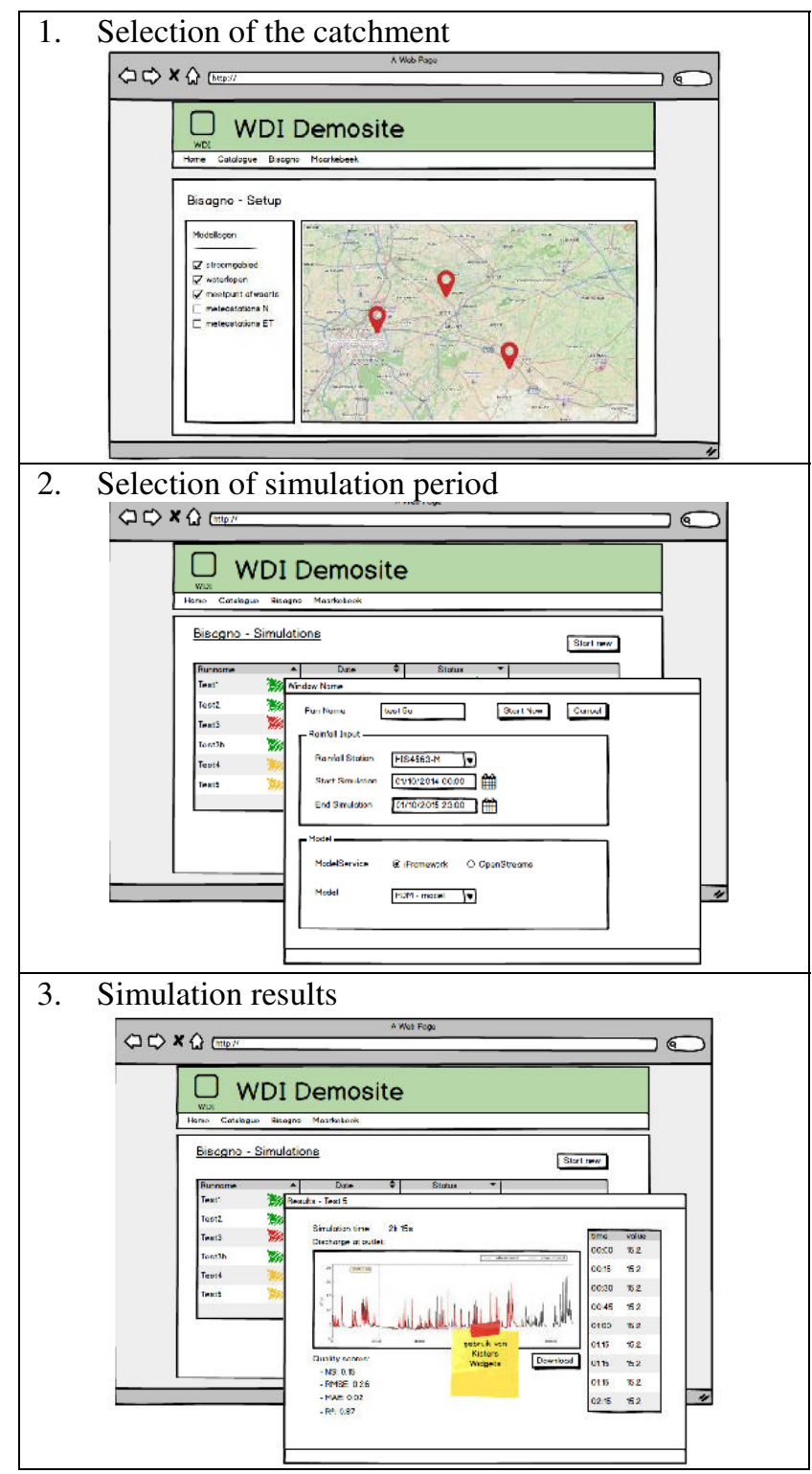

Figure 8: Mockups of the user interaction of the FHMenvironment web application

\section{Conclusions}

Flanders Environment Agency wants their modelling tools and forecasting system on the long term to evolve towards layered, web-enabled, flexible, modular, open and standardized components and services. To improve the applicability of models, it is important to move to a flexible and modular system where models can be combined and easily interchanged in order to deliver relevant results for different applications and on a variety of temporal and spatial scales. In a first step the development of a Flanders Hydrological Model environment was initiated. This model environment offers modellers a simple, flexible and open software environment, integrated with the Python-PCRaster system. For rapid use it includes a set of predefined model codes as well as model components, calibration algorithms, and statistics. The appeal of the system lies in the ability to fluidly transform data, by passing it through chains of functions from the database to the expected output. The steps are fully automated and can quickly be extended by defining new modules. This should enable more innovative developments resulting in an enhanced hydrological model performance and the integration of such models into services useful for broader applications.

In a first application a Flemish regional Hydrological Model was built with the model environment. This large scale model - still under development - includes a combination of sub-models, mutually linked within the model environment. Each submodel describes the hydrological behavior of a catchment. Four sub-models in different hydro-geological regions of Flanders were highlighted. The simulation results show that flow observations are simulated fairly well. All four models reproduced the catchment runoff with high efficiencies (NSE $>0.72$ ). Volumes and also hydrographs are simulated with acceptable matching capabilities. This Flemish Hydrological Model can on the long term be a reference for authorities involved in water management and to support policy making.

A second application demonstrated the use of relatively recent techniques to integrate the modelling framework into a web environment. This web service application allows the modelling framework to be used by other platforms and a broader range of clients. At the user end, the aim is to provide a user-friendly interface for allowing the user to interact with the modelling framework through the web. Anyone can discover, access and run the hydrological modelling framework. Hereto, protocols were implemented for data retrieval and conversion, but also for output data visualization. At the server side, the aim is to provide seamless links with other platforms or data stores. It was developed in such a way to allow for easy integration into new platforms. Web applications have the potential to share data, provide easy access for users with limited modelling knowledge, and assemble data and information customized for specific topics that support informed policy-making. Hereto, there is need to develop more web-based tools to make sharing of datasets and information available. When datasets, modelling systems and tools would be available as web services in an integrated space, a modeller can plug in the data and link the model components that are needed to make a reliable simulation. Combined with real-time data and online computations, this opens the way for interactive modelling. Water authorities, however, have limited expertise and experience with these technologies. As the modelling framework is open, it is the intention that further methodological innovation and associated developments will come from a wider community, including science, technology and operations. Such structural collaboration within a community is also needed for continuously enhancing the performance of the modelling framework through innovation. The 
modelling framework should be continuously refined and improved to account for the best available modelling concepts. The modelling framework is flexible enough to implement new modules to improve aspects whose results are unsatisfactory to the end user. Additional modules could be new routing schemes such as the Muskingum method for routing floods events, additional calibration procedures or optimization routine (e.g. DREAM), modules to support uncertainty estimation based on parameter sampling. They can also focus on external extra-disciplinary processes, such as erosion, groundwater, nutrient leaching, etc. Anyway, the Flanders Hydrological Model environment might give an boost to hydrological modelling in Flanders.

\section{Acknowledgements}

This work was supported by the Flanders Environment Agency, Ministry of Environment, Nature and Energy, Flanders, Belgium. We acknowledge all research institutes and consultants that worked on the development of the model environment. Likewise, the experts of the steering committee are acknowledged for their valuable remarks.

\section{References}

1 Moore, R.J., 1985. The probability-distributed principle and runoff production at point and basin scales. Hydrological Sciences Journal, 30, 273-297.

2 Moore, R.J., 2007. The PDM rainfall-runoff model. Hydrology and Earth System Sciences, 11, 1, 483499.

3 Nielsen, S.A., Hansen, E., 1973. Numerical simulation of the rainfall-runoff process on a daily basis. Nordic Hydrology, 4, 171-190.

4 Abbott, M.B., Bathurst, J.C., Cunge, J.A., Oconnell, P.E., Rasmussen, J., 1986a. An Introduction to the European Hydrological System - Système Hydrologique Européen, She. 1. History and Philosophy of A Physically-Based, Distributed Modeling System. Journal of Hydrology, 87, 45-59.

5 Abbott, M.B., Bathurst, J.C., Cunge, J.A., Oconnell, P.E., Rasmussen, J., 1986b. An Introduction to the European Hydrological System - Système Hydrologique Européen, She. 2. Structure of A Physically-Based, Distributed Modeling System. Journal of Hydrology, 87, 61-77.

6 Arnold, J.G., Srinivasan, R., Muttiah, R.S., Williams, J.R., 1998. Large area hydrologic modeling and assessment part I : Model development. Journal of American Water Research Association, 34, 1, 73-89

7 Beven, K.J., Kirkby, M.J., 1979. A Physically Based Variable Contributing Area Model of Basin Hydrology. Hydrology Sciences Bulletin 24, 43-69.

8 Jakeman, A.J., Littlewood, I.G., Whitehead, P.G. 1990. Computation of the instantaneous unit hydrograph and identifiable component flows with application to two small upland catchments. Journal of Hydrology, 117, 275-300.
9 Cole, S.J., Robson, A.J., Bell, V.A., Moore, R. J.: Model initialisation, data assimilation and probabilistic flood forecasting for distributed hydrological models, in: Geophysical ResearchAbstracts, 11, EGU2009-8048-3, 2009.

10 Cabus, P., 2008. River flow prediction through rainfall-runoff modeling with a probabilitydistributed model (PDM) in Flanders, Belgium. Agricultural Water Management, 95, pp. 859-868

11 Dewelde, J., Verbeke, S., Quintelier, E., Cabus, P., Vermeulen, A., Vansteenkiste, T., De Jongh, I., Cauwenberghs, K., 2014.Real-time flood forecasting in Flanders. 11th International Conference on Hydroinformatics HIC 2014, New York City, USA; Conference proceedings.

12 Van Steenbergen, N., Willems, P., 2012. Method for testing the accuracy of rainfall- runoff models in predicting peak flow changes due to rainfall changes, in a climate changing context. Journal of Hydrology, 414-415, 425-434.

13 Van Steenbergen, N., Willems, P., 2014. Quantification of rainfall forecast uncertainty and its impact on flood forecasting. 11th International Conference on Hydroinformatics - HIC 2014, New York City, USA.

14 Breuer, L., Huisman, J.A., Willems, P., Bormann, H., Bronstert, A., Croke, B.F.W., Frede, H.-G., Gräff, T., Hubrechts, L., Jakeman, A.J., Kite, G., Lanini, J., Leavesley, G., Lettenmaier, D.P., Lindström, G., Seibert, J., Sivapalan, M., Viney, N.R., 2009. Assessing the impact of land use change on hydrology by ensemble modeling (LUCHEM) I: Model intercomparison with current land use. Advances in Water Resources, 32 (2), 129-146.

15 Viney, N.R., Bormann, H., Breuer, L., Bronstert, A., Croke, B.F.W., Frede, H., Gräff, T., Hubrechts, L., Huisman, J.A., Jakeman, A.J., Kite, G.W., Lanini, J., Leavesley, G., Lettenmaier, D.P., Lindström, G., Seibert, J., Sivapalan, M., Willems, P., 2009. Assessing the impact of land use change on hydrology by ensemble modelling (LUCHEM) II: Ensemble combinations and predictions. Advances in Water Resources, 32 (2), 147-158.

16 Ludwig, R., May, I., Turcotte, R., Vescovi, L., Braun, M., Cyr, J.-F., Fortin, L.-G, Chaumont, D., Biner, S., Chartier, I., Caya, D., Mauser, W., 2009. The role of hydrological model complexity and uncertainty in climate change impact assessment. Advanced. Geosciences, 21, 63-71.

17 Maurer, E.-P., Brekke, L.-D., Pruitt, T., 2010. Contrasting lumped and distributed hydrology models for estimating climate change impacts on California watersheds. Journal of the American Water Resources Association, 46 (5), 1024-1035.

18 Van Steenbergen, N., Ronsyn, J., Willems, P., 2012. A non-parametric data-based approach for probabilistic flood forecasting in support of uncertainty communication. Environmental Modelling \& Software, 33, 92-105

19 Vansteenkiste, T. , Tavakoli, M., Ntegeka, V., De Smedt, F., Batelaan, O., Pereira, F., Willems, P., 2014. Intercomparison of hydrological model 
structure and calibration approaches in climate scenario impact projections. Journal of Hydrology, 519A, 27, 743-755.

20 Werner, M., Cranston, M., Harrison, T., Whitfield, D., Schellekens, J., 2009. Recent developments in operational flood forecasting in England, Wales and Scotland. Meteorololgical Applications, 16, 13-22.

21 De Lange, W.J., Prinsen, G.F, Hoogewoud, J.C, Veldhuizen, A.A, Verkaik, J., Oude Essink, G.H.P., van Walsum P.E.V., Delsam, J.R, Hunink, J.C., Massop, H.Th.L., Kroon, T., 2014. An operational, multi-scale, multi-model system for for consensusbased, integrated water management and policy analysis: The Netherlands Hydrological Instrument. Environmental Modelling \& Software, 59, 98-108.

22 Martens, B., Cabus, P., De Jongh, I., Verhoest, N.E.C., 2013. Merging weather radar observations with ground-based measurements of rainfall using an adaptive multiquadric surface fitting algorithm. Journal of Hydrology, 500, 84-96

23 Karssenberg, D. De Jong, K, Van der Kwast, J., 2007. Modelling landscape dynamics with Python. International Journal of Geographical Information Science, 19, 623-637.

24 PCRaster, January 2013. PCRaster internet site. Available online at: http://www.python.org

25 Willems, P., 2014. Parsimonious rainfall-runoff model construction supported by time series processing and validation of hydrological extremes Part 1: Step-wise model-structure identification and calibration approach. Journal of Hydrology, 510 (14), 578-590.

26 Willems, P., Quan, T.Q, Van den Zegel, B., De Decker, K., Pannemans, B., Gullentops, C. Buitrago, S., Blanckaert, J., Adams, R., 2013. Next Generation Tool for Flexible Hydrological Modelling - concept note. Concept-eindrapport. KU Leuven \& IMDC, project L 2012 T 0001 X Perceel 2 Dijle / Vlaamse Milieumaatschappij - Afdeling Operationeel Waterbeheer, maart 2014.

27 Leavesley, G.H., Restrepo, P.J., Stannard, L G., Frankoski, L.A., Sautins, A.M., 1996. The modular modeling system (MMS) - A modeling framework for multidisciplinary research and operational applications. GIS and environmental modeling: Progress and research issues, M. Goodchild et al., eds., GIS World Books, Fort Collins, Colorado,155158.

28 Kraft, P., Vache, K.B., Frede, H.-G., Breuer, L., 2011. A hydrological programming language extension for integrated catchment models. Environmental Modelling and Software, 26, 828830.

29 Fenicia, F., Savenije, H.H.G., Matgen, P., Pfister, L., 2008. Understanding catchment behavior through stepwise model concept improvement. Water Resources Research 44, W01402p

30 Duan, Q.Y., Gupta, V.K., Sorooshian, S., 1993. Shuffled complex evolution approach for effective and efficient global minimization. Journal of Optimization Theory and Applications, 76 (3), 501521.
31 Tran, Q.Q., Willems, P., Pannemans, B., Blanckaert, J., Pereira, P., Nossent, J., Cauwenberghs, K., Vansteenkiste, T., 2015. Flexible hydrological modeling - Disaggregation from lumped catchment scale to higher spatial resolutions. Geophysical Research Abstracts 17, EGU2015-6983-1.

32 Nash, J.E., Sutcliffe, J.V., 1970. River flow forecasting through conceptual models, I, A discussion of principles. Journal of Hydrology, 10, 282-290.

33 Buytaert, W., Baez, S., Bustamante, M., Dewulf, A., 2012. Web-Based Environmental Simulation: Bridging the Gap between Scientific Modeling and Decision-Making. Environmental science \& technology, 46, 1971-1976.

34 Alberti, K., de Jong, K., Karssenberg, D., A virtual globe for environmental impact assessment. European Geosciences Union, EGU General Assembly, 2014.

35 Bröring, A., Maué, P., Janowicz, K., Nüst, D., Malewski, C., 2011. Semantically-Enabled Sensor Plug \& Play for the Sensor Web. Sensors, 11 (8), 7568-7605.

36 Maué P., Stasch, C., Athanasopoulos, G., Gerharz, L., 2011. Geospatial Standards for Web-enabled Environmental Models. Internal Journal for Spatial Data Infrastructures Research (IJSDIR), 6.

37 Wolfs, V., Meert, P., Willems, P., 2015. Modular conceptual modelling approach and software for river hydraulic simulations. Environmental Modelling \& Software 71, 60-77. 